\title{
MISUSE OF THE AXIOM OF INDEPENDENCE In the ANalytic Hierarchy Process
}

\author{
David Hauser \\ Joseph M. Katz Graduate School of Business, 247 Mervis Hall \\ University of Pittsburgh, Pittsburgh, Pennsylvania 15260, U.S.A.
}

Kirti Peniwati

Institute for Management Education and Development

Jl. Menteng Raya 9, Jakarta 10340, Indonesia

\begin{abstract}
Some authors have proposed that the Analytic Hierarchy Process (AHP) axiom of independence be relaxed to accommodate observations drawn from: a) examples of pairwise comparisons of alternatives in clusters in single criterion AHP problems, and b) examples of problems in which the criteria have the same underlying measurement, and both the achievement of the goal and the alternatives are measured objectively. We show that the illustrations given are actually single criterion problems according to the AHP. Thus the AHP axiom of independence is inapplicable in both situations and therefore not violated. We also consider the consequence of failure to distinguish between a criterion as

- an antibute of alternatives and a cluster of alternatives, the two being different in the hierarchic structure. Finally, we discuss transformable problems, which look like multicriteria problems but are actually single criterion problems and how failure to recognize this fact may lead to incorrect syntheses and false conclusions.
\end{abstract}

Keywords - Analytic Hierarchy Process, decision making, multicriteria decision making, clustering.

\section{Introduction}

The Analytic Hierarchy Process (AHP), has received widespread attention from both academics and practitioners. As of this writing, there have been over 1,000 publications and diverse applications in business and government institutions [7,10]. However, there remains some confusion as to how this deceptively simple and intuitive methodology works [4]. For example, some authors $[9,11]$ have advanced various arguments and suggestions to change hierarchic composition by focusing on artificial clustering and manipulation down the hierarchy and concluded that these operations should influence the priorities of the criteria back up the hierarchy. Because the AHP axiom of independence prohibits that, they propose that this axiom be relaxed to accommodate their observations. Their arguments are derived from examples using absolute measurement, in which incorrect outcomes (sometimes reversing rank) are observed when the AHP axiom of independence is applied.

The axiom of independence cannot be relaxed, otherwise one would not be able to establish priorities in cause/effect relations in which the causes are perceived to be independent of their effects. Independence is an essential assumption for dealing with practical problems. The AHP does in fact aiso provide the theory for dealing with the dependence of criteria on alternatives by employing a more general network structure $[5,6,7]$ with its supermatrix. Our analysis shows that none of the conventional justifications suggested by a variety of authors $[9,11]$ can improve the AHP on how it is now applied with its axiom of independence.

We will discuss four main themes:

1) Clusters versus criteria, in which we discuss the functional, structural, and 
mathematical distinctions between the two.

2) Comparison versus manipulation, in which we show examples of improper manipulation of measurements.

3) Criteria versus criterion, in which we study how some "multicriteria" problems are actually single criterion AHP problems and we demonstrate that the axiom is thus inapplicable.

4) Multicriteria and multiscales, in which we emphasize the problems of an over reliance on absolute numbers.

By forcing the application of the axiom inappropriately, one will mistakenly conclude that there is a problem with the axiom rather than with understanding the definition of the problem. Examples from the literature are brought fourth to make our point. Thus we will show the logical weakness in the arguments made by these authors to relax the axiom, and conclude that their proposal to change it is highly questionable.

\section{Mechanical Clusters, Not Criteria}

The AHP methodology requires one to compare a small number of homogeneous elements to improve consistency of the judgments $[5,6,7]$. A large number of alternatives must be divided into clusters with a small number of elements, by a method we call mechanical clustering. Clustering of this sort is an arbitrary grouping of homogenous elements, the manner of which does not influence the final priorities of the alternatives. Mechanical clustering differs from routine mechanical clustering which applies to inhomogeneous elements and which group elements into small homogenous sub-sets with linking elements. One must not confuse mechanical clustering with the definition of criteria in a hierarchy. The axiom of independence only applies to the latter because it creates new elements (criteria) which by themselves must be pairwise compared, hence will influence the priority of the alternatives. In general, criteria contribute to the goal in a manner different than mechanical clusters which have no creative information content to express the transmission of influence in the hierarchy.

Confusing mechanical clusters for criteria, Wedley et al. [11] applied the axiom of independence to mechanical clusters. They applied the AHP in a single criterion problem of estimating the relative distance of various cities from Singapore, clustered according to their general direction from Singapore. The results were validated when the authors normalized the actual distance data. Next, they deleted one city from each cluster and recalculated the relative distance of the cities without changing the cluster weights which should have been updated but were not. They then incorrectly concluded that the AHP axiom of independence needed to be relaxed so that weighting normalized alternative weights by the old cluster weight and by the new cluster weight give the same answer. This was an arithmetic manipulation, rather than a generalizable concept about setting priorities.

Since their conclusion is based on the assumption that criteria and mechanical clusters are synonymous, we must point out that there are actually three main distinctions to be made between these two concepts.

\section{FUNCTIONAL DISTINCTION}

If mechanical clusters can be treated as criteria, their removal should have precisely the same impact as the removal of criteria. Suppose we delete all the mechanical clusters from a 
hierarchy with clusters, we will still pairwise compare the alternatives with respect to the goal in the usual manner. The only impact of removing the clusters would be that we cannot take advantage of the decreased number of paired comparisons. If mechanical clusters are the same as criteria, we should find the same impact if we remove the criteria from a hierarchy with criteria. However we have fewer comparisons to make. Similarly, the questions asked for the judgments have changed. In the criteria-less hierarchy, we pairwise compare the alternatives relative to the goal, not to a criterion. This indicates that the removal of criteria will increase the crudeness of the judgments. Thus the removal of criteria and the removal of mechanical clusters do not have the same impact on their respective problems.

To illustrate, consider the following two examples. First, suppose we wish to evaluate several employees for a promotion based on four criteria, (computer skills, attitude/motivation, leadership skills, and quality of work), where the employees are named Jeffrey, David, and Lisa. See Figure 1. If we delete the four criteria we obtain the hierarchy of

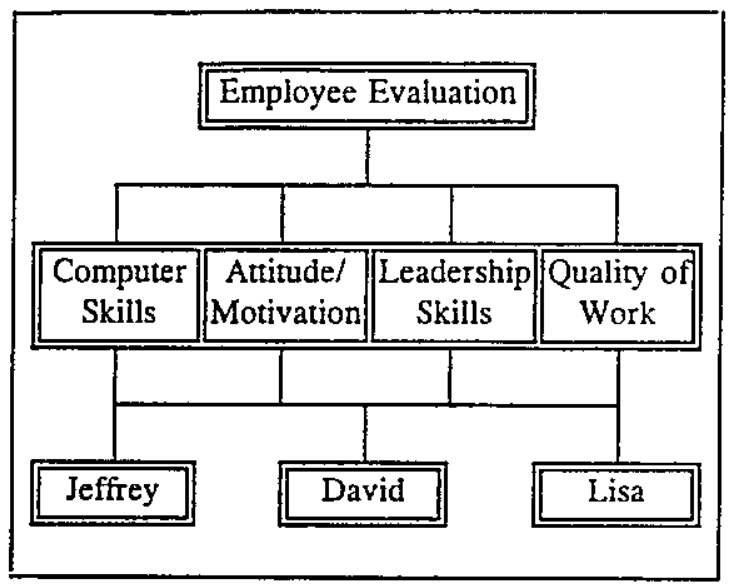

Figure 1 -- Employee Evaluation Model.

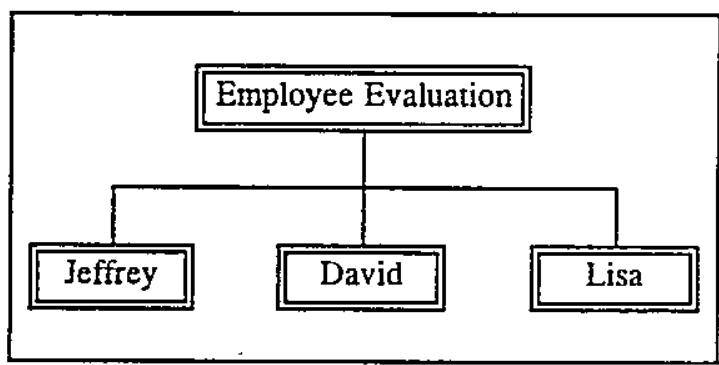

Figure 2 -- First altered form of the Employee Evaluation Model. Figure 2, in which we can no longer meaningfully compare the alternatives. Thus the criteria play a functional role in the synthesis of alternative weights

However, suppose we wish to determine the relative areas of a set of shapes. For simplicity, we clustered the alternatives into similar shapes. See Figure 3. If we delete the clusters, we obtain the hierarchy in Figure 4. When we pairwise compare the alternatives, we still pairwise compare them relative to the goal. Thus the clusters do not play a functional role in the synthesis of their alternatives weights. Therefore mechanical clusters cannot be treated as criteria.

\section{STRUCTURAL DISTINCTION}

Suppose we construct a hierarchy composed of a goal, a set of mechanical clusters, and a set of alternatives. The only questions asked of the decision maker in this structure is: "with respect to the goal, which altemative is dominant over which other alternative, and by how much?" However these pairwise comparison questions only relate those alternatives mechanically clustered together. Suppose then that we interchange the position of the alternatives level and the

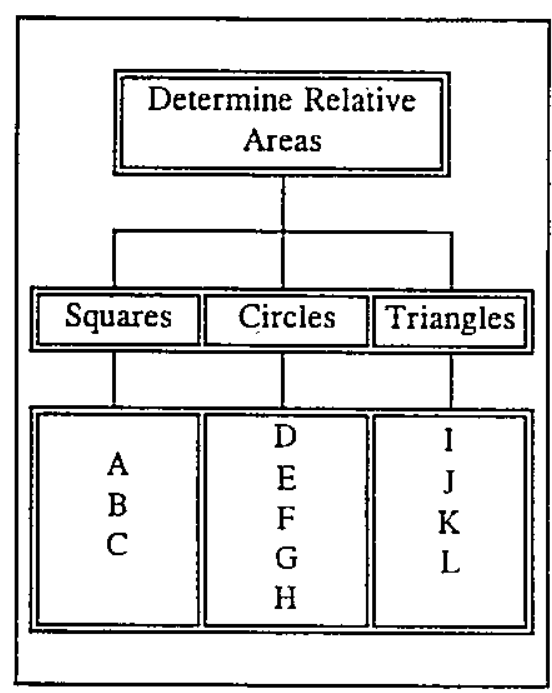

Figure 3 -- Area Model. mechanical clusters level. By design, we know that the mechanical clusters are not a functional 
part of the hierarchy, rather they are a simplifying part of the structure. Therefore the original hierarchy and this second altered form of the cluster-hierarchy should not differ in synthesized alternative weights. We know this will hold since we must now pairwise compare the alternatives in one large comparison matrix with respect to the goal, hence the same questions will be asked. The mechanical clusters do not change the logic or data required regarding the alternatives.

In contrast, suppose we construct a hierarchy composed of a goal, a set of criteria, and a set of alternatives. There are two sets of questions asked of the decision maker in this structure: 1) "with respect to the goal, which criterion is dominant over which other criterion, and by how much?" and 2) "with respect to criterion $i$, which alternative is dominant over which other alternative, and by how much?" for all i. Suppose then that we interchange the position of the alternatives level and the criteria level. By design, we know that the criteria are a functional part of the hierarchy aiding in the differentiation of the alternatives. Therefore one must expect that the questions asked regarding the alternatives should no longer be the same. In the second altered form of the cluster-hierarchy, we again ask two sets of questions of the decision maker: 1) "with respect to the goal, which alternative is dominant over which other alternative, and by how

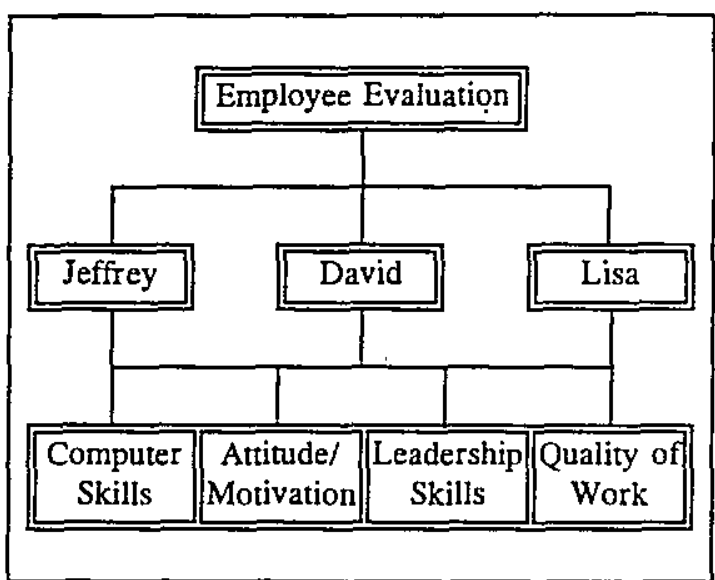

Figure 5 -- Second altered form of the Employee Evaluation Model. much?" and 2) "with respect to alternative i, which criterion is dominant over which other, and by how much?"

To illustrate, consider once again the two examples of Figures 1 and 3 . In the employee evaluation model, by interchanging the criteria level and the alternatives level, the outcome changed from ranking employees to ranking skills. See Figure 5. This indicates that one must have changed the goal, hence we cannot be dealing with the same problem as we did at the start. By interchanging the cluster level and the alternatives level in the area model, the original outcome (and the original goal) remains intact. See Figure 6. In the updated employee evaluation problem, one of the questions is: "with respect to Jeffrey, which skill is more dominant and by how much?" The overall priorities of this updated version of the problem would indicate which attribute is most dominant across all employees of the company. In the original version of the problem, the employees are in competition with one another, whereas in this structurally altered problem, the employees together distribute these attributes throughout the company and

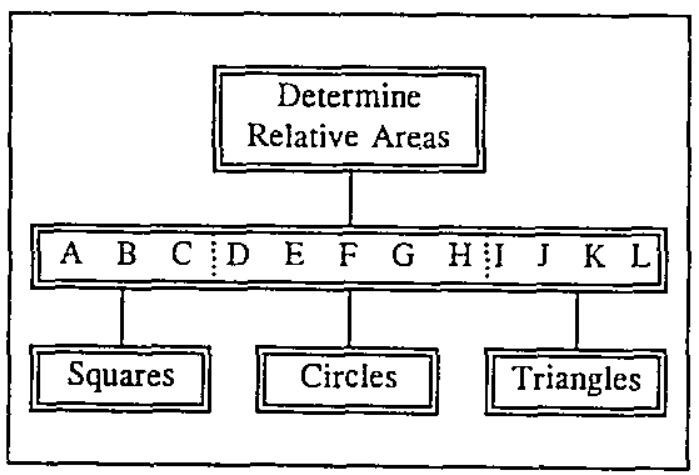

Figure 6 -- Second altered form of the Area Model. 
hence the final synthesis indicates which attribute is most prevalent in the firm. In contrast, no new information is required or created in the updated area model when the clusters level and the alternatives level were interchanged. The function of the clusters remains constant regardless of the structural change, whereas for criteria, the function of the criteria changes with a change in the structure. Therefore we note that as levels of a hierarchy, the alternatives are structurally dependent on criteria, while the alternatives are structurally independent of clusters. Thus one cannot claim that mechanical clusters are equivalent to criteria.

\section{MATHEMATICAL DISTINCTION}

When we are presented with a set of criteria which help differentiate the alternatives, we must pairwise compare them to determine relative dominance with respect to the goal. However, the weights of mechanical clusters must be derived, not from comparisons, but from the structural and functional dependence they have on the problem.

Consider once again the mechanical cluster hierarchy in which the clusters level and aiternatives level were interchanged. We can synthesize the weights of the clusters. Therefore we can develop an argument for the dependence of cluster weights on alternative weights. Suppose $\mathrm{n}$ homogeneous alternatives are grouped into $\mathrm{k}$ mechanical clusters, where each cluster contain $n_{j}$ alternatives, $j=1, \ldots, k$. Let $\alpha_{j}$ be the weight of mechanical cluster $j$, and $w_{i j}$ be the global weight of alternative $i$ of cluster $j, i=1, \ldots, n, j=1, \ldots, k$, derived from pairwise comparing all $n$ alternatives in one matrix, where the vector $w=\left\{w_{i j}\right\}$ is the non-trivial solution of $A w=n w$, with respect to cluster $j$, and let $y_{i j}$ be the local weight of the $i^{\text {th }}$ alternative with respect to the $\mathrm{j}^{\text {th }}$ cluster.

The weight of any element is passed to any element below. Therefore the global weight of element $\mathrm{i}$ of level $\mathrm{k}$ is equal to the local weight of element $\mathrm{i}$ of level $\mathrm{k}$ times the global * weight of its governing element from level $\mathrm{k}-\mathrm{i}$. We know that

$$
w_{i j}=\sum_{j=1}^{n} y_{i j} \alpha_{j}
$$

In clustering without the use of a common element, the $\mathrm{i}^{\text {th }}$ alternative appears in only one cluster, and thus equation (1) can be simplified to

$$
w_{i j}=y_{i j} \alpha_{j}
$$

Consider then the two feasible cases regarding the number of clusters:

Case 1: $\quad \mathrm{k}=1$.

This is equivalent to one comparison matrix containing all, $n$ elements. Therefore the

local weight of the $i^{\text {th }}$ alternative must equal the global weight of the $i^{\text {th }}$ alternative.

Case 2: $\quad \mathrm{k}>1$

From (1) and (2), we know that $\alpha_{\mathrm{j}}$ is a function of $\mathrm{w}_{\mathrm{ij}}$ and $\mathrm{y}_{\mathrm{ij}}$. We have already determined that $w_{i j}$ is a constant. However, as $k$ changes or as $n_{j}$ changes, only $y_{i j}$ changes and thus $\alpha_{\mathrm{j}}$ must change accordingly such that equations (1) and (2) still hold.

In sum, we note that the global weight of alternative $i$ is independent of the cluster to which it is assigned, and therefore it is independent of the number of clusters and of the number 
of alternatives within a cluster.

\section{Comparison, Not Manipulation}

Schoner and Wedley [9] were concerned with a car selection problem employing the objective of minimizing cost over an intended usage period where the extent of usage per year is known. The alternative cars were compared with respect to purchase price, maintenance cost per year, and fuel consumption in gallons per mile. They computed scale factors for each criterion to convert each of the three different criteria measurements to the single unit (dollars) used in the objective. This scheme permits one to add the converted measurements describing each alternative to obtain a total score which is in the units of the objective, therefore one could take the minimum of these total dollar values as defined by the objective. These operations cannot be meaningfully employed in AHP multicriteria problems because the theory assumes that the criteria have different relative ratio scales. Thus one may argue at this stage that the AHP is either not applicable to this kind of problem, or that the problem needs to be carefully ordered to be compatible with AHP relative measurement. We will show how to do this.

Schoner and Wedley contend that this problem requires that one add each alternative's scaled intensity ratings with respect to each criterion to arrive at a composite measure, then compare the alternatives. They assume that it' is a multicriteria AHP problem, hence argue that applying the AHP methodology should result in the same answer as the "optimal" solution derived by direct calculation. The definition of optimality for a particular problem is defined by the objective of that problem. As such, the "optimal" alternative in the car selection problem is the one with the lowest $\mathrm{v}_{\mathrm{i}}$ obtained from

$$
v_{i}=\sum_{k} q_{k} \cdot T_{i k}
$$

where $q_{k}$ is a scale factor which converts a measurement on attribute $\mathrm{k}$ to units of the objective, and $\mathrm{T}_{\mathrm{ik}}$ represents the absolute measurement of option $\mathrm{i}$ on criterion or attribute $\mathrm{k}$. To arrive at relative ratios, we must map $v_{i}$ to a $[0,1]$ ratio scale, hence

$$
w_{i}=\frac{\sum_{k} q_{k} \cdot T_{i k}}{\sum_{i, k} q_{k} \cdot T_{i k}}
$$

where $w_{i}$ is the composite priority of option $i$ as used by the authors [9]. Equation (4) suggests that for one to compute the ratios that correspond to the optimal solution, one must normalize the absolute intensity ratings of each alternative after obtaining the total of the absolute intensity ratings with respect to each criterion. One must also conclude that to ensure arithmetic compatibility between all criteria, one must find a scaling factor for each criterion such that the product of the scaling factor and the unit of its corresponding criterion results in the same unit as the objective.

They use the following arithmetically relation: 


$$
x_{k}=(K)\left(q_{k}\right)\left(\sum_{h} T_{h k}\right)
$$

where $\mathrm{K}$ is a constant of proportionality, and $\Sigma_{\mathrm{h}} \mathrm{T}_{\mathrm{hk}}$ is the sum of the absolute measurements of the alternatives with respect to each criterion to conclude that "....the relative importance of a criterion must be proportional to the product of its scaling factor and the sum (or average) of the absolute values of option measurements on that criterion." Despite the argument that criteria weights are dependent on $\Sigma_{h} \mathrm{~T}_{h k}, w_{i}$ is independent of $\Sigma_{h} \mathrm{~T}_{h k}$. In reality, $\Sigma_{h} \mathrm{~T}_{\mathrm{hk}}$ influences the criteria weights simply because the authors normalized the absolute data for each criterion as a consequence of assuming that this problem is a multicriteria AHP problem. Then the issue becomes whether or not their formula is generally meaningless.

Consider an example given by the authors [9], where a firm must choose between two products, where the two relevant criteria represent sales in two different markets, as follows:

\begin{tabular}{|c|c|c|c|c|}
\hline Option & Criterion 1 & Criterion 2 & Sum & Ratio of Sums \\
\hline A & 100 & $3 \mathrm{~s}$ & $100+3 \mathrm{~s}$ & $(100+3 \mathrm{~s}) /$ \\
B & 200 & $\mathrm{~s}$ & $200+\mathrm{s}$ & $(200+\mathrm{s})$ \\
\hline
\end{tabular}

Clearly here, the ratio computed using local ratios suggested by the authors cannot be equivalent to the ratios of the synthesized weights. We note the similarity of this problem with the car selection problem in that this problem also dictates that one convert the composite absolute measurements into a $[0,1]$ ratio scale, rather than convert the absolute scale of each criterion into a $[0,1]$ ratio scale. Because of the indigenous relationship between the two criteria (indicated by the existence of formulas to describe their relationship with the data), it is wrong to assume that one can independently judge that the two criteria are equally important. Assuming that one can do so, and applying the AHP, the authors get a different answer from the one given by their arithmetic manipulation. This lead Schoner and Wedley [9] to question whether the AHP yields ratio-scale measurements.

The authors reason that to apply the AHP, criteria importance $\left(\pi_{k}\right)$ must be measured by:

$$
\pi_{k}=\mathrm{K} \cdot \mathrm{q}_{\mathrm{k}} \cdot \mathrm{T}_{\mathrm{k}} \cdot
$$

where $T_{k}{ }^{*}$ represents the absolute measurement of the largest value option under criterion $k$ [9], as proposed by Belton and Gear [1,2]. The authors [9] apply the AHP ideal mode of relative measurement, in which the local priority vector is normalized in such a way that the highest priority alternative with respect to a given criterion receives a local weight of unity and all other alternatives have proportionately smaller values [7]. Again, independence is nowhere violated. It is not clear, however, why the authors use different formulas for criteria importance in the car selection problem and in the product selection problem. In fact, one could argue that the ideal mode of relative measurement is more appropriate for the car selection problem (provided that the problem is structured as a multicriteria AHP problem) because it is more likely that irrelevant options will be added into the set of alternatives, from which the decision maker may want to preserve previous rank.

Using the same argument as in the car problem, we must conclude that $\mathrm{T}_{\mathrm{x}}{ }^{*}$ came into the 
formula because the authors normalized the absolute measurements of the alternatives with respect to each criterion (as required by the AHP) rather than normalizing the composite absolute measurements across all the criteria measured in that absolute scale, committing the same arithmetic error in turning absolute scales of measurements into relative scales of measurement in a piecemeal way.

In both examples, the weights of the criteria were computed mechanically from the absolute (objective) data. In general, the criteria weights do not reflect the willingness of the decision maker to make a trade off between the criteria as intended by the AHP since the preference of the decision maker is not considered in the formula. Rather than concluding that the weights of the criteria are dependent on the totals (or averages) of the measurements of all the alternatives, it would have been more appropriate to conclude that these problems cannot be solved meaningfully by converting the original scales of the criteria into normalized ratio scales. Moreover, the fact that scaling factors are also included in the formula indicates that their argument is not generalizable to multicriteria problems involving different absolute measurement scales (let alone those that have no measurement).

\section{Criterion, Not Criteria}

To synthesize absolute measurements in a meaningful manner, Saaty [7] said "...we must normalize the factors measured with respect to a single scale..." Forman [3] wrote, "the [car selection] example [(Schoner and Wedley, 1989)] ... is really a single criterion problem, the criterion being dollars." Here are some of the hidden assumptions made in arithmetically using an absolute scale: 1) the criteria share the same (or can be mapped with a scaling factor to the same) underlying unit, 2) the perception of the decision maker of the common unit is constant across all mapped criteria, 3) the alternatives are homogenous with respect to each criterion, and 4) the alternatives can be individually measured with some absolute scale or intensity rating with respect to each criterion. Call this class of problems transformable problems. For these problems, the optimal solution can be obtained by combining all intensity ratings as dictated by the objective [9]. We will discuss how transformable problems, which appear as multicriteria problems, are in fact single criterion problems in the AHP.

Suppose we are interested in choosing a car based solely on final purchase price. In this instance, it is clear that the alternative with the lowest absolute measurement with respect to price is the best choice. Suppose then we dissected the final purchase price criterion into initial purchase price and tax. Since the tax is a constant fraction of the purchase price, we know that the absolute measurements for each alternative with respect to tax will be a constant fraction of the initial purchase price of each alternative. Since the initial price and tax price are measured on the same scale and that we perceive $l$ unit precisely the same way on each criterion, one should be able to treat the seemingly "multicriteria" version of the car problem as a single criterion. In order to combine these transformable criteria, they must share the same underlying unit and we must perceive one unit precisely the same way with respect to each criterion.

To complicate matters, suppose our car example included average fuel consumption as another criterion. Since average fuel consumption is not in the same unit as the others, we cannot necessarily assume that it is transformable. However, since each unit of fuel consumption is linearly correlated with the dollar, we can multiply each alternative's 
measurement with respect to average fuel consumption by $\mathrm{x}$ (dollar*miles) / gallon, and thus fuel consumption is transformable to the unit of the objective. We scale each fuel consumption measurement into dollars and note that $\$ 1$ can be perceived the same way in this scaled version of the criterion as we perceive $\$ 1$ in the initial price or the tax price. Therefore all three criteria are transformable. However, suppose we add a fourth criterion, cabin size. It may be that cabin size is exponentially correlated with its cost. Therefore if we multiply it by a constant, the new scale is likely to be meaningless. Nonetheless, suppose we do multiply each measurement of cabin size by y dollars per cubic inch. We cannot assume that $\$ 1$ in this newly scaled criterion is perceived the same way as $\$ 1$ in all of the other mapped criteria. Therefore it is meaningless to convert this criterion into dollars, hence we conclude that cabin size is not transformable relative to the unit of the objective.

Schoner and Wedley's [9] formula is not generalizable to nontransformable problems. One must combine each group of transformable criteria meaningfully, then use the resulting combined criterion in the AHP hierarchy in the traditional manner. Saaty [7] discusses this conclusion and demonstrates how to appropriately aggregate such criteria in the multicriteria problem. In multicriteria-like problems such as the car selection example given by Wedley and Schoner, the decision maker does not have to consider trade offs between the criteria, so the problem is analogous to the single criterion problem in which no trade offs are made among the criteria (given that single criterion problems only consider one criterion).

Based on the above discussion, we must conclude that the author's [9] car selection problem is transformable and thus we must concur with Forman [3]. To apply the AHP, they should have first combined all transformable criteria into a single criterion. Since their problem is a single criterion AHP problem, the axiom of independence cannot be violated.

Care must be taken to meet the entire definition of a transformable problem, otherwise erroneous conclusions can be drawn. For instance, suppose each element of a set of criteria shared the same unit of measurement along an absolute scale. One cannot conclude then that these criteria can necessarily be combined. For example, both criteria of a two criteria problem may be in (or mapped to) dollars. However one criterion may be described as a cost-like criterion and the other may be described as a benefit-like criterion. Therefore the perception of one unit (or one dollar in this case) must be questioned. Only if the perception of $\$ 1.00$ is precisely the same across both transformed-criteria can this problem be considered a single criterion problem. If one were considering a problem where it is illogical or irrelevant to the goal to pairwise compare some or all of the criteria, this too would not imply that those criteria are transformable. It may be that these elements are not homogeneous such as the cost-like criterion and the benefit-like criterion $[5,6,7]$.

\section{Multicriteria and Multiscales}

Values are context dependent, therefore they cannot be converted to a single universal unit that is applicable in all contexts. We might surmise that some people such as economists and utility theorists would have us convert all these diverse units into dollars. Even if we could, a unit of a dollar is different in size and rate of change from context to context. At the end, we have to deal with our own contingent priority scales. Consider the following more realistic car selection problem in which we include several tangible and intangible criteria. 


\begin{tabular}{||c|c|c|c|c|}
\hline \multirow{2}{*}{ Alternatives } & \multicolumn{4}{|c|}{ Criteria } \\
\cline { 2 - 5 } & Price & Maintenance & Space & Comfort \\
\hline Car A & $\$ 10,000$ & $\$ 5,000$ & $32 \mathrm{cu} . \mathrm{ft}$ & High \\
Car B & $\$ 15,500$ & $\$ 4,000$ & $25 \mathrm{cu} . \mathrm{ft}$ & Fair \\
Car C & $\$ 20,000$ & $\$ 2,000$ & $20 \mathrm{cu} . \mathrm{ft}$ & Low \\
\hline
\end{tabular}

Despite our argument that values are context dependent, let us assume that price and maintenance can be combined using their numerical data, hence the formula proposed by Schoner and Wedley [9] applies. Now, how can we deal with the other criteria? Although measurable, it is difficult to accept that the unit of space can be converted into dollars. Let us assume however, although it may not be realistic, that we are able to convert cubic feet into dollars. How can we compute the scale factor for this criterion? The scale factor is supposed to be a constant across all alternatives for a given criterion. But realistically we know that the decision maker's preference may not be linear with respect to space. The optimal size for the decision maker is not necessarily the biggest or the smallest cabin. Must we insist that the judgments be consistent with the formula, otherwise the decision maker would make a wrong decision? How do we determine the scaling factor? The problem becomes even worse when one has to deal with intangibles like comfort that has no measurement. What would be the scaling factor for this criterion? Assuming that somehow one can compute such a factor, the next question would be: how are we going relate price and maintenance cost which are transformable, space which has a different scale, and comfort which does not have an objective measurement? Given these unanswered questions, we cannot generally convert the unit of each criterion into the unit of the objective using the proposed arithmetic approach and then combine with intangible factors since the conversion approach is not generalizable. Hence we must first combine all transformable criteria and then apply the traditional comparison method to derive a meaningful scale.

\section{Conclusion}

It has been shown that applying AHP mechanics in hierarchies which violate the AHP axiom of independence results in misleading conclusions and improper expectations. We have shown two considerable points of confusion regarding the AHP: 1) mistaking mechanical clusters of alternatives (clusters that do not provide additional meaning to the hierarchy) for criteria (elements which do provide additional meaning to the hierarchy), and 2) mistaking transformable AHP problems for multicriteria AHP problems. Both of these errors lead to faulty proposals to modify the AHP. We have shown that the need to pairwise compare alternatives in clusters, does not automatically add new criteria. We have also shown that because an AHP problem looks like a multicriteria problem does not imply that it is a multicriteria AHP problem.

The AHP requires that the relative importance of criteria should not be determined by the measurement of the alternatives, although it does not necessarily follow that the decision maker need never know anything about the alternatives. It is part of the decision maker's learning process so that (s)he understands the context of the problem to be able to construct a hierarchy representing and to make judgments for the relative importance of the criteria. 
Above all, the AHP is not simply about manipulating absolute numbers and assuming that the correct outcome is the one that is consistent with the numbers derived from arithmetic manipulation. The AHP is about comparing decision elements, not manipulating hard data. Priorities provide the nonlinear cushion to transform units into other units in the context of each individual problem. It is rarely meaningful to assume that we have a universal unit to which all other measurements can be converted.

\section{APPENDIX}

Definition: Let $A=\left(a_{i j}\right)$ be an $(n+1)$ by $(n+1)$ consistent positive reciprocal matrix. Let $A^{\prime} \subset A$ where $A^{\prime} \equiv A$ with the $u^{\text {th }}$ row and $u^{\text {th }}$ column of $A$ removed. Let $w$ be the non-trivial solution of $A w=(n+1) w$, and $w^{\prime}$ be the non-trivial solution of $A^{\prime} w^{\prime}=n w^{\prime}$.

Theorem A: The normalized components of $w$ with the $u^{\text {th }}$ element deleted equals $w$ '.

Proof: $\quad$ By definition of consistency, we know w', which satisfies $A^{\prime} w^{\prime}=n w^{\prime}$, must equal any column of $A^{\prime}$ normalized, and $w$, which satisfies $A w=(n+1) w$, must equal any column of $A$ normalized. Hence we can express $w$ ' and $w$ as

$$
w^{\prime}=\left\{\frac{a_{i j}}{\sum_{i=1}^{n} a_{i j}}\right\} \quad j=1, \ldots, n
$$

and

$$
w=\left\{\frac{a_{i j}}{\sum_{i=1}^{n+1} a_{i j}}\right\} \quad j=1, \ldots, n+1
$$

The denominators of the components of w' and $w$ are constants and the numerator of the $j^{\text {th }}$ component of $w^{\prime}$ is equal to the $j^{\text {th }}$ component of $w$, $\mathrm{j}=1, \ldots, \mathrm{n}$.

Theorem B: The rank of a set of alternatives is preserved when a new alternative is added and the updated reciprocal matrix $A=\left(a_{i j}\right)$ is consistent.

Proof: $\quad$ Since rank is preserved when we compare the entire $w$ with the first $n$ elements of ' $w$ ', the rank cannot change when comparing the first n elements of vector $w$ ' with the entire vector $w$. 


\section{References}

[1] Belton, V., and A. E. Gear, "On a Shortcoming of Saaty's Method of Analytic Hierarchies," Omega, v11n3, 1983.

[2] Belton, V., and A. E. Gear, "The Legitimacy of Rank Reversal--A Comment," Omega, v13n3, 1985.

[3] Forman, E. H., "AHP is Intended for More Than Expected Value Calculations," Decision Sciences, v21, 1990, pp. 670-2.

[4] Forman, E. H., "Facts and Fictions about the Analytic Hierarchy Process," Mathematical and Computer Modelling, v14n4-5, 1993, pp. 19-26.

[5] Saaty, T. L., The Analytic Hierarchy Process, New York, New York, McGraw-Hill Book Company, Inc., 1980.

[6] Saaty, T. L., Multicriteria Decision Making: The Analytic Hierarchy Process, RWS Publications, Pittsburgh, 1990.

[7] Saaty, T. L., Fundamentals of Decision Making and Priority Theory, RWS Publications, Pittsburgh, 1994.

[8] Saaty, T. L., and L. G. Vargas, "Inconsistency and Rank Preservation," Journal of Mathematical Psychology, v28n2, June 1984.

[9] Schoner, B. and W. C. Wedley, "Ambiguous Criteria Weights in AHP: Consequences and Solutions", Decision Sciences, 20, 1989, pp.462-475.

[10] Vargas, L. G., "An Overview of the Analytic Hierarchy Process and its Applications," European Journal of Operational Research, v48n1, 1990, pp. 2-8.

[11] Wedley, W. C., Schoner, B., and E. U. Choo, "Clustering, Dependence and Ratio Scales in AHP: Rank Reversals and Incorrect Priorities with a Single Criterion", Journal of Multi-criteria Decision Analysis, 2, 1993, pp. 145-158. 